\title{
Evaluation of suitable spectral intervals for near-IR laboratory detection of water vapour continuum absorption
}

Article

Accepted Version

Ptashnik, I. V. (2007) Evaluation of suitable spectral intervals for near-IR laboratory detection of water vapour continuum absorption. Journal of Quantitative Spectroscopy \& Radiative Transfer, 108 (1). pp. 146-160. ISSN 0022-4073 doi: https://doi.org/10.1016/j.jqsrt.2007.03.011 Available at https://centaur.reading.ac.uk/853/

It is advisable to refer to the publisher's version if you intend to cite from the work. See Guidance on citing.

To link to this article DOI: http://dx.doi.org/10.1016/j.jgsrt.2007.03.011

Publisher: Elsevier Ltd.

Publisher statement: Copyright @ 2007 Elsevier Ltd

All outputs in CentAUR are protected by Intellectual Property Rights law, including copyright law. Copyright and IPR is retained by the creators or other copyright holders. Terms and conditions for use of this material are defined in the End User Agreement.

www.reading.ac.uk/centaur 
Central Archive at the University of Reading

Reading's research outputs online 


\title{
Evaluation of suitable spectral intervals for near-IR laboratory detection of water vapour continuum absorption
}

\author{
Igor V. Ptashnik \\ Laboratory of Atmospheric Absorption Spectroscopy, \\ Institute of Atmospheric Optics, Akademichesky 1, Tomsk, 634055, Russia \\ E-mail: piv@iao.ru, Fax: (007) 3822492086
}

\begin{abstract}
The water vapour continuum absorption is an important component of molecular absorption of radiation in atmosphere. However, uncertainty in knowledge of the value of the continuum absorption at present can achieve $100 \%$ in different spectral regions leading to an error in flux calculation up to $3-5 \mathrm{~W} / \mathrm{m}^{2}$ global mean. This work uses line-by-line calculations to reveal the best spectral intervals for experimental verification of the $\mathrm{CKD}$ water vapour continuum models in the currently least studied near-infrared spectral region. Possible sources of errors in continuum retrieval taken into account in the simulation include the sensitivity of laboratory spectrometers and uncertainties in the spectral line parameters in HITRAN-2004 and Schwenke-Partridge database. It is shown that a number of micro-windows in near-IR can be used at present for laboratory detection of the water vapour continuum with estimated accuracy from 30 to $5 \%$.
\end{abstract}

\section{Introduction}

The water vapour continuum absorption (henceforth 'continuum'), which varies slowly with wavelength, is an essential component of molecular absorption of radiation in the atmosphere contributing up to $5-6 \%$ to the total absorption of solar radiation in near IR spectral region. The semi-empirical continuum model - CKD [1], which is used today by most of atmospheric radiation transfer codes, until recently defined the continuum absorption coefficient as follows: 


$$
\begin{aligned}
& k_{\mathrm{c}}(v)=R(v, T) \cdot \sum_{i}\left\{S_{i}(T)\left[f_{\mathrm{c}}\left(v-v_{i}\right) \chi^{\prime}\left(v-v_{i}\right)+f_{\mathrm{c}}\left(v+v_{i}\right) \chi^{\prime}\left(v+v_{i}\right)\right]\right\}, \\
& f_{\mathrm{c}}\left(v \pm v_{i}\right)= \begin{cases}\frac{1}{\pi} \cdot \frac{\gamma_{i}}{\Delta v_{\text {cutoff }}^{2}+\gamma_{i}^{2}}, & \left|v \pm v_{i}\right| \leq \Delta v_{\text {cutoff }} \\
\frac{1}{\pi} \cdot \frac{\gamma_{i}}{\left(v \pm v_{i}\right)^{2}+\gamma_{i}^{2}}, & \left|v \pm v_{i}\right|>\Delta v_{\text {cutoff }}\end{cases}
\end{aligned}
$$

where $v$ is wavenumber $\left(\mathrm{cm}^{-1}\right)^{*} ; R(v, T)$ is a radiation field term, accounting for the effects of stimulated emission; $S_{i}(T)$ is intensity of spectral line $i ; \gamma_{i}$ is Lorentzian halfwidth; $f_{\mathrm{c}}\left(v+v_{i}\right)$ is continual part of the spectral line; $\Delta v_{\text {cutoff }}$ characterizes the spectral interval near line centre where the local contribution of the spectral line absorption is accounted for (see Fig. 1). In the CKD model $\Delta v_{\text {cutoff }}=25 \mathrm{~cm}^{-1}$. The parameters of the $\chi^{\prime}\left(v \pm v_{i}\right)$ - function are derived in the CKD model by fitting to experimental data.

The recent versions of the CKD continuum model - CKD-2.4 [2] and MT_CKD [3] (http://rtweb.aer.com/continuum_frame.html) use some different physical interpretation and parameterisation of the function $k_{\mathrm{c}}(v)$. Apart from far wings contribution of allowed transitions, described by (1), a term describing absorption due to collision-induced transitions was added. However, the line profile that should be used with the MT_CKD continuum model is still described by Voigt profile without the $25 \mathrm{~cm}^{-1}$ basement (see Fig. 1).

The successful parameterisation used in CKD continuum model has made it widely adopted in many atmospheric applications. Using only one type of line profile for all lines and few adjustable parameters the CKD model seems to provide reasonable agreement with experiment over wide spectral regions. However, it has been shown by some investigations that the CKD continuum model still contains essential uncertainty, which becomes apparent when comparing different versions of the model, reaching in some spectral regions $100 \%$ and causing difference in calculated absorption of solar radiation up to $2.5-3 \mathrm{~W} / \mathrm{m}^{2}$ [4-6]. It is clear that disagreement between the model and real continuum absorption can be even more. This is especially the case in the near-IR and visible spectral regions, because so far line shape parameters in the CKD model have been derived by fitting to experiments mostly in the middle and far infrared region (i.e. $\left.>4 \mu \mathrm{m}\left(0-2500 \mathrm{~cm}^{-1}\right)\right)$ [7-10], and have not been thoroughly verified at shorter wavelengths. Precise measurements of water vapour continuum could also clarify still disputable question about its nature; in particular, the possible water dimer contribution to the self-continuum that was first suggested in [11,12] and discussed recently in [13-15].

\footnotetext{
* The author uses ' $v$ ' throughout the paper without traditional tilde which is officially applied for wavenumber in order to distinguish it from the frequency.
} 
Thus, the problem of experimental verification of the CKD continuum model in near-IR and visible spectral regions is still very topical. Until recently it was a very difficult experimental challenge because of the very weak continuum absorption in this spectral range. It is seen from the Fig. 2 that water continuum decreases rapidly with wavenumber and does not exceed $10^{-6}-10^{-7} \mathrm{~cm}^{-1}$ in near-IR band wings at room conditions.

So far very few successful works are known on water vapour continuum measurements in near-IR and visible region. In the work [16] laboratory measurements of continuum absorption were carried out in micro-windows (i.e. in areas between spectral lines) in the water vapour absorption band 3000-4200 $\mathrm{cm}^{-1}$. In papers [17] and [18] laboratory measurements of continuum absorption were made at fixed wavelengths $1.056 \mu \mathrm{m}$ and 0.6943 $\mu \mathrm{m}$ respectively $\left(9466 \mathrm{~cm}^{-1}\right.$ and $\left.14399 \mathrm{~cm}^{-1}\right)$. In both cases the relative error was estimated to be about $30 \%$. Finally, the report on water vapour measurement on the atmospheric slant path in the absorption bands $0.72 \mu \mathrm{m}\left(13900 \mathrm{~cm}^{-1}\right)$ and $0.94 \mu \mathrm{m}\left(10600 \mathrm{~cm}^{-1}\right)$ [19] as well as laboratory measurements in the band $1.89 \mu \mathrm{m}\left(5300 \mathrm{~cm}^{-1}\right)$ [13] appeared recently. Results of all these investigations confirm the thesis about significant uncertainty of CKD continuum model in the short-wave spectral region. Measurement [17] and [18] demonstrated respectively about $70 \%$ and $30 \%$ excess of the measured continuum (mostly self-continuum in [17] and foreign-continuum in [18]) over prediction of the MT_CKD model. Foreigncontinuum absorption registered in [19] in the absorption band $0.94 \mu \mathrm{m}$ was found to be a factor of 2 and 1.5 less than in the CKD-2.4 and MT_CKD models respectively. In the 0.72 $\mu \mathrm{m}$ band the measured foreign-continuum appeared to be in a good agreement with the CKD2.4 model, but it was a factor of 1.6 higher than in the MT_CKD model. An excess of the measured self-continuum absorption by factor 1.5 over the CKD-2.4 model was found also in [13].

Significant progress achieved during the past 10 years in experimental laser spectroscopy allows however considerable extension of the evaluation of the CKD model in the near-IR region. The methods of photoacoustic and ring-down spectroscopy can provide sensitivities up to $10^{-9}-10^{-10} \mathrm{~cm}^{-1}$. The present work uses numerical simulation to reveal the best spectral intervals in the near-IR for laboratory measurements of water vapour continuum. The results obtained here can be used also for the field measurements if absorption by water vapour is not saturated in the chosen spectral interval for the given path length. However, the difficulty in determining water vapour amount and aerosol contribution in the field conditions often does not allow achieving reasonable accuracy in retrieving water continuum absorption. 


\section{Numerical simulation and analysis}

To search for spectral intervals optimal for detecting the water vapour continuum in laboratory conditions the calculations of absorption spectra were performed for a mixture of water vapour with air and for pure water vapour in the region $2500-15000 \mathrm{~cm}^{-1}(0.67-4 \mu \mathrm{m})$. For calculations we used line-by-line code reported in [20]. Results of the simulation for the air-broadened case in the whole near-IR and in chosen spectral intervals are presented in the Figs. 3-7. Simulation was made for 20 mbar water vapour with 993 mbar synthetic air (i.e., $80 \% \mathrm{~N}_{2}$ and $20 \% \mathrm{O}_{2}$ ) to exclude the possible contribution of $\mathrm{CO}_{2}, \mathrm{O}_{3}$ and $\mathrm{CH}_{4}$, which, however, is not matter of great importance near water vapour band centres. The simulation is performed for the temperature $296 \mathrm{~K}$.

The upper part of each panel of the figures shows water vapour lines absorption spectrum with and without MT_CKD continuum absorption taken into account $\left(K_{\text {Lines+MT_CKD }}\right.$ and $K_{\text {Lines }}$ respectively). The both cases imply cutting off line profile within $25 \mathrm{~cm}^{-1}$ from the line centre. The smooth curves $\left(K_{\mathrm{MT}}\right.$ CKD and $\left.K_{\mathrm{CKD}-2.4}\right)$ present separately MT_CKD and CKD-2.4 continuum. Water vapour line absorption $\left(K_{\text {Lines }}\right)$ was calculated using the SchwenkePartridge linelist [21] with parameters of the strong lines replaced from HITRAN-2004 [22]. This retains the higher accuracy of the stronger spectral lines parameters from HITRAN-2004 compared to the Schwenke-Partridge linelist, but allows the many weak lines absent in HITRAN. This approach, suggested to the author by B. A. Fomin, was discussed in details in $[5,6]$.

The lower part of each panel shows the ratio of the MT_CKD continuum absorption to the water vapour lines absorption without continuum (i.e. $K_{\mathrm{MT}+\mathrm{CKD}} / K_{\text {Lines }}$ ), and the ratio $K_{\mathrm{MT} \_\mathrm{CKD}} / \Delta K$, where $\Delta K$ is an estimated absolute value of the total error (experimental and computational) in the supposed water vapor continuum retrieval. This value includes: a) possible systematic error of measurements $\delta_{\text {sys, }}$, which assumed here to be 0.03 (an example of systematic error estimation can be found elsewhere [23]); b) random error of measurement, determined by noise level $k_{\text {noise }}$ of the detector (assumed here to be $2 \cdot 10^{-9} \mathrm{~cm}^{-1}$ ); c) uncertainty in accounting for the local contribution of spectral lines, caused by inaccurate knowledge of the spectral lines parameters in HITRAN-2004. The later accounted for uncertainty indices (so called "error codes") presented in HITRAN for line centres, intensities, air- and selfbroadened widths, pressure shifts and temperature dependencies. Thus, the error $\Delta K(v)$ was estimated as follows: 


$$
\Delta K(v)=\left[\sum_{i=1}^{6} \Delta k_{i}(v)^{2}+k_{\text {noise }}^{2}\right]^{1 / 2}+\delta_{\text {sys }} K_{\text {Lines }+ \text { MT_CKD }},
$$

where $\Delta k_{i}$ is deviation of the calculated absorption coefficient from the original value ( $K_{\text {Lines+MT_CKD }}$ ) caused by changing one of the six line parameters by the value of uncertainty of this parameter in HITRAN-2004. For the weak lines from the Schwenke-Partridge linelist [21], that do not have explicit information about uncertainty of their parameters, the error of intensities, broadening coefficients and temperature dependencies were assumed to be equal $30 \%$, and uncertainty in line centres of $0.2 \mathrm{~cm}^{-1}$.

Figures 8-13 show similar results for 20 mbar pure water vapour case (self-broadening).

Summarizing the plotted values it can be mentioned that the ratio $K_{\mathrm{MT}+\mathrm{CKD}} / K_{\mathrm{Lines}}$ characterizes the "detectivity level" for continuum absorption as compared to the Lorentzian line absorption, whereas the ratio $K_{\mathrm{MT}} \mathrm{CKD}_{\mathrm{C}} / \Delta K$ can be considered as a "signal to noise" equivalent (or inverse relative error of the continuum retrieval). Thus, spectral areas with $K_{\mathrm{MT} \text { CKD }} / \Delta K \geq 2$ or 3 (i.e., where the expected relative error of the derived continuum is less than $\sim 50-30 \%$ ) may be regarded as the 'good' ones for experimental continuum evaluation. The $K_{\mathrm{MT}}{ }_{-} \mathrm{CKD} / K_{\text {Lines }}$ value, on the other hand, can be useful to estimate impact of possible additional errors in line parameters in HITRAN-2004 and Schwenke-Partridge database on the retrieval of the water vapour continuum. It is clear that the higher $K_{\mathrm{MT}} \mathrm{CKD}_{\mathrm{C}} / K_{\mathrm{Lines}}$ ratio corresponds to lesser sensitivity of the retrieved continuum value to the uncertainty in parameters of the subtracted spectral lines.

The presented figures show that there are a number of micro-windows in the spectral intervals $0.719-0.73 \mu \mathrm{m}\left(13700-13900 \mathrm{~cm}^{-1}\right), 0.815-0.832 \mu \mathrm{m}\left(12020-12260 \mathrm{~cm}^{-1}\right), 0.893-$ $0.98 \mu \mathrm{m}\left(10200-11200 \mathrm{~cm}^{-1}\right), 1.11-1.15 \mu \mathrm{m}\left(8650-9000 \mathrm{~cm}^{-1}\right), 1.28-1.5 \mu \mathrm{m}\left(6700-7800 \mathrm{~cm}^{-}\right.$ $\left.{ }^{1}\right), 1.69-2.1 \mu \mathrm{m}\left(4800-5900 \mathrm{~cm}^{-1}\right)$ and 2.2-2.5 $\mu \mathrm{m}\left(3900-4550 \mathrm{~cm}^{-1}\right)$, where significant contribution of the continuum absorption is expected $\left(K_{\mathrm{MT}}{ }_{\mathrm{CKD}} / K_{\mathrm{Lines}} \geq 0.5\right)$ and this absorption exceeds by at least a factor of 3 the possible errors of measurements with photoacoustic or cavity ring-down spectrometers $\left(K_{\mathrm{MT}} \mathrm{CKD}_{\mathrm{C}} / \Delta K>3\right)$.

Using lower value of the systematic error $\left(\delta_{\text {sys }}\right)$ or/and higher detector sensitivity (i.e. lower random noise $k_{\text {noise }}$ ) in specific measurements may allow an increase in the ratio $K_{\mathrm{MT}} \mathrm{CKD}_{\mathrm{C}} / \Delta K$ in particular spectral regions. It is found, however, that the main contribution to the total error $\Delta K(v)$ (formula (2)) in the perspective for continuum evaluation intervals is caused by uncertainties in line parameters in HITRAN-2004 \& Schwenke-Partridge database. Most important are line intensities, broadening coefficients and line centre positions. It means 
that the relative error of the continuum retrieval can be decreased (i.e. $K_{\mathrm{MT}}{ }_{\mathrm{CKD}} / \Delta K$ in Figs. 313 increased) by experimentalists by preliminary fitting of the line parameters to measured spectrum, which may allow correcting them compared to the values presented in HITRAN \& Schwenke-Partridge database ${ }^{\dagger}$. This emphasizes the advantage of high spectral resolution measurements (as compared to the low resolution) even for detection of the continuum absorption.

\section{Conclusion}

A simple approach is suggested to evaluate the spectral intervals most suitable for the water vapour continuum detection in the near-IR region. The approach accounts for possible systematic and random errors of experiment and for uncertainty in spectral line parameters in the modern databases. It is shown that a number of micro-windows located mainly within water vapour absorption bands and their close wings can be used at present for laboratory detection of the continuum absorption with accuracy from 30 to 5\%. Most of these spectral regions overlap the areas of generation of diode lasers and other laser sources and thus can be used for the measurements. Many of the found intervals can also be applied for detection of the continuum on the basis of Fourier transform spectrometer with multipath cell providing optical path length up to $1000 \mathrm{~m}$, with sensitivity achieving $10^{-8} \mathrm{~cm}^{-1}$.

Despite the relative simplicity of the approach presented above and to certain degree evidence of the main conclusion the author hopes that the results reported here will stimulate additional experiments on water vapour continuum detection in near-infrared spectral region.

\section{Acknowledgements}

The author would like to thank K. Firsov and K. Shine for useful comments. The work was supported by Russian Foundation for Basic Research (Grant No. 04-05-64569a) and in part by Scientific School "Optical spectroscopy of molecules and radiative processes in atmosphere" (Project RI-112/001/020).

\footnotetext{
$\dagger$ The simultaneous fitting of the line parameters together with the local baseline fitting is required to avoid partial including of the continuum absorption into the line strength and halfwidth parameters [24].
} 


\section{References}

1. Clough SA, Kneizys FX, Davies RW. Line shape and water vapour continuum. Atmos Res $1989 ; 23: 229-241$.

2. Mlawer EJ, Clough SA, Brown PD, Tobin DC. Recent developments in the water vapour continuum. Ninth ARM Science Team Meeting Proceedings, March 22-26, 1999, San Antonio, TX, p.1-6.

3. Mlawer EJ, Tobin DC, Clough SA. A revised perspective on the water vapour continuum: The MT_CKD model. JQSRT, in preparation.

4. Zhong W, Haigh JD, Belmiloud D, Schermaul R, Tennyson J. Note on 'The impact of new water vapour spectral line parameters on the calculation of atmospheric absorption'. QJR Meteorol Soc 2002;128:1387-1388.

5. Ptashnik IV, Shine KP. Calculation of solar radiative fluxes in the atmosphere: the effect of updates in spectroscopic data. Atmos Oceanic Opt 2003;16(3):251-255.

6. Fomin BA, Udalova TA, Zhitnitskii EA. Evolution of spectroscopic information over the last decade and its effect on line-by-line calculations for validation of radiation codes for climate models. JQSRT 2004;86:73-85.

7. Birch DE, Alt RL. Continuum absorption in the $700-1200 \mathrm{~cm}^{-1}$ and $2400-2800 \mathrm{~cm}^{-1}$ windows. Rep. AFGL-TR-84-0128 (U.S. Air Force Geophysics Laboratory), 1984.

8. Tobin DC, Strow LL, Lafferty WJ, Olson WB. Experimental investigation of the self- and $\mathrm{N}_{2}$-broadened continuum within the $v_{2}$ band of water vapour. Appl Opt 1996;35:47244734 .

9. Han Y, Shaw JA, Churnside JH, Brown PD, Clough SA. Infrared spectral radiance measurements in the tropical Pacific atmosphere. J Geophys Res 1997;102:4353-4356.

10. Tobin DC, Best FA, Brown PD, Clough SA, Dedecker RG, Ellingson RG, Garcia RK, Howell HB, Knuteson RO, Mlawer EJ, Revercomb HE, Short JF, van Delst PF, Walden VP. Down-welling spectral radiance observation at SHEBA ice station: Water vapour continuum measurements from 17-26 $\mu \mathrm{m}$. J Geophys Res 1999;104: 2081-2092.

11. Penner SS and Varanasi P. Spectral absorption coefficients in the pure rotation spectrum of water vapour. JQSRT 1967; 7: 687-690.

12. Varanasi P, Chou S, Penner SS. Absorption coefficients for water vapour in the 600-1000 $\mathrm{cm}^{-1}$ region. JQSRT 1968; 8: 1537-1541.

13. Ptashnik IV, Smith KM, Shine KP, Newnham DA. Laboratory measurements of water vapour continuum absorption in spectral region $5000-5600 \mathrm{~cm}^{-1}$ : Evidence for water dimers. QJR Meteorol Soc 2004;130:2391-2408. 
14. Daniel JS, Solomon S, Kjaergaard HG, Schofield DP. Atmospheric water vapour complexes and the continuum. Geophys Res Letters 2004;31:L06118.

15. Cormier JG, Hodges JT, Drummond JR. Infrared water vapour continuum absorption at atmospheric temperatures. J Chem Phys 2005;122:114309.

16. Birch DE. Absorption by $\mathrm{H}_{2} \mathrm{O}$ in narrow windows between $3000-4200 \mathrm{~cm}^{-1}$. Rep. AFGLTR-85-0036 (U.S. Air Force Geophysics Laboratory), 1985.

17. Fulghum SF, Tilleman MM. Interferometric calirometer for the measurement of watervapour absorption. J Opt Soc Amer 1991;8B:2401-2413.

18. Tikhomirov AB, Ptashnik IV, Tikhomirov BA. Photoacoustic measurements of the water vapour continuum absorption in the $14400 \mathrm{~cm}^{-1}(0.694 \mu \mathrm{m})$ wavenumber region. Optics and Spectroscopy 2006;101(1):80-89.

19. Sierk B, Solomon S, Daniel JS, Portmann RW, Gutman SI, Langford AO, Eubank CS, Dutton EG, Holub KH. Field measurements of water vapour continuum absorption in the visible and near-infrared. J Geophys Res 2004;109:D08307.

20. Mitsel' AA, Ptashnik IV, Firsov KM, Fomin BA. Efficient technique for line-by-line calculating the transmittance of the absorbing atmosphere. Atmos Oceanic Opt $1995 ; 8(10): 847$.

21. Schwenke DW, Partridge H. Convergence testing of the analytic representation of an abinitio dipole moment function for water: Improved fitting yields improved intensities. J Chem Phys 2000;113:6592-6597.

22. Rothman LS, Jacquemart D, Barbe A, D. Chris Benner, Birk M, Brown LR, Carleer MR, Chackerian C Jr., Chance K, Dana V, Devi VM, Flaud J.-M, Gamache RR, Goldman A, Hartmann J.-M, Jucks KW, Maki AG, Mandin J.-Y, Massie ST, Orphal J, Perrin A, Rinsland CP, Smith MAH, Tennyson J, Tolchenov RN, Toth RA, Vander J Auwera, Varanasi P, Wagner G. The HITRAN 2004 Molecular Spectroscopic Database. JQSRT 2005;96(2):139-204.

23. Coheur P.-F, Fally S, Carleer M, Clerbaux C, Colin R, Jenouvrier A, Merienne M.-F, Hermans C, Vandaele AC. New water vapour line parameters in the $26000-13000 \mathrm{~cm}^{-1}$ region. JQSRT 2002;74:493-510.

24. Ptashnik IV, Smith KM, Shine KP. Self-broadened line parameters for water vapour in the spectral region 5000-5600 $\mathrm{cm}^{-1}$. J Mol Spectrosc 2005;232(2):186-201. 


\section{Figure captions}

Figure 1. Solid and dashed lines schematically show respectively continual $\left(f_{\mathrm{c}}\right)$ and spectral line $\left(f_{\text {line }}\right)$ contribution of the line profile according to the CKD continuum model.

Figure 2. CKD-2.4 and MT_CKD-1.0 water vapour continuum models are presented for atmospheric conditions (20 mbar $\mathrm{H}_{2} \mathrm{O}$, ambient pressure 1013 mbar, $296 \mathrm{~K}$ ). Highresolution water vapour absorption spectrum, simulated using HITRAN-2004 database and MT_CKD continuum, is shown by grey background for comparison.

Figures 3. The upper part of every panel shows simulated water vapour absorption spectrum for 20 mbar water vapour in 993 mbar synthetic air with and without MT_CKD-1.0 continuum taken into account ( $K_{\text {Lines+MT_CKD }}$ and $K_{\text {Lines }}$ respectively). Water vapour line absorption $\left(K_{\text {Lines }}\right)$ is calculated using Schwenke-Partridge linelist with parameters of the strong lines replaced from HITRAN-2004. The lower part of the panels shows the ratio of the continuum absorption $K_{\mathrm{MT} \text { CKD }}$ to the water lines contribution $K_{\text {Lines }}$, and to the estimated total error $\Delta K$ of the continuum retrieval.

Figures 4-7. Same as in fig. 3, but in the spectral regions chosen as most promising for continuum detection.

Figures 8. Same as in fig. 3, but for 20 mbar pure water vapour case.

Figures 9-13. Same as in fig. 8, but in the spectral regions chosen as most promising for continuum detection. 
Fig. 1

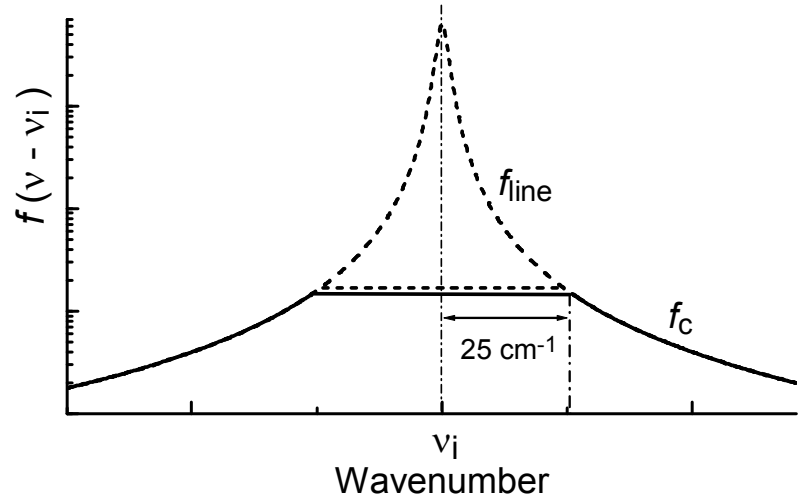

Fig. 2

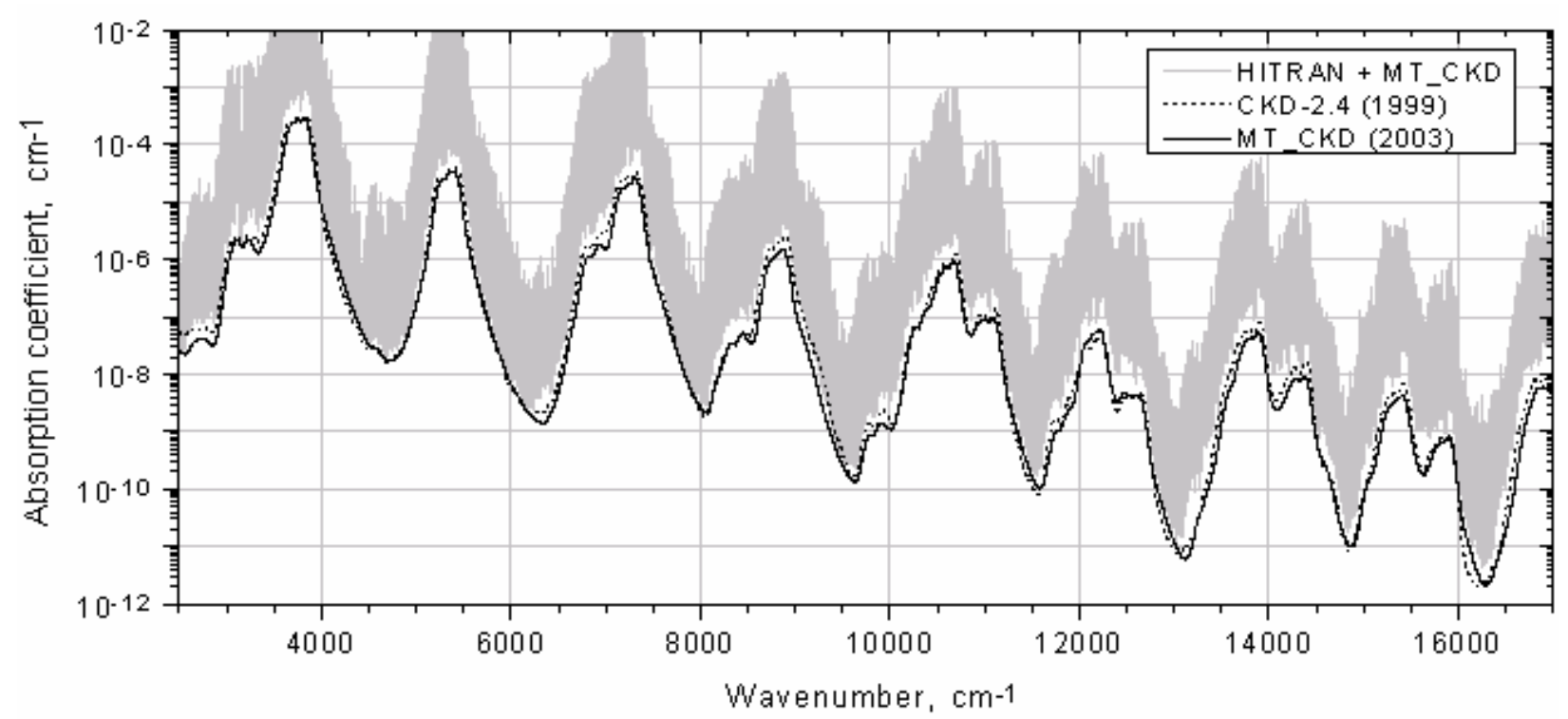

Fig. 3

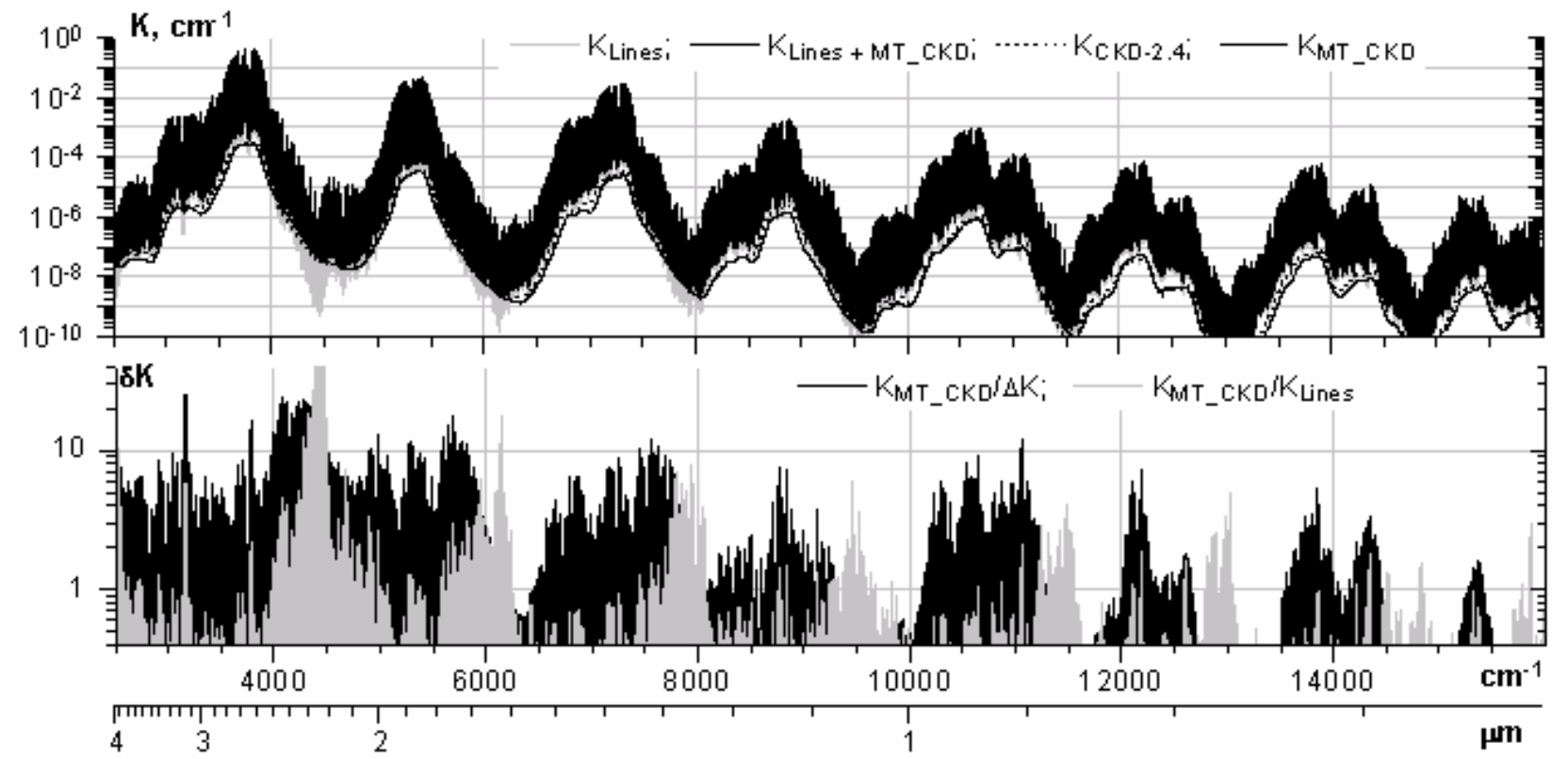


Fig. 4
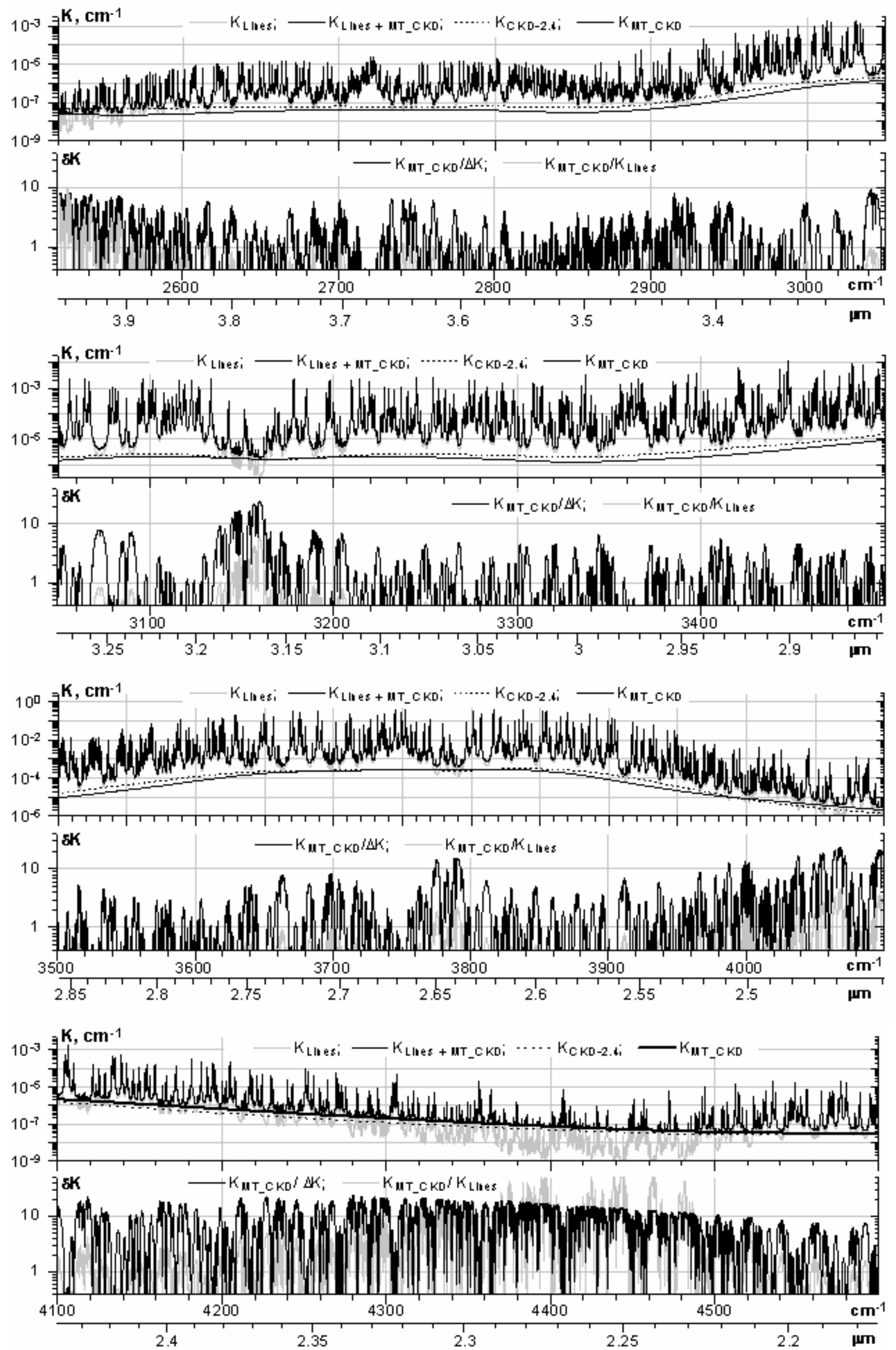
Fig. 5
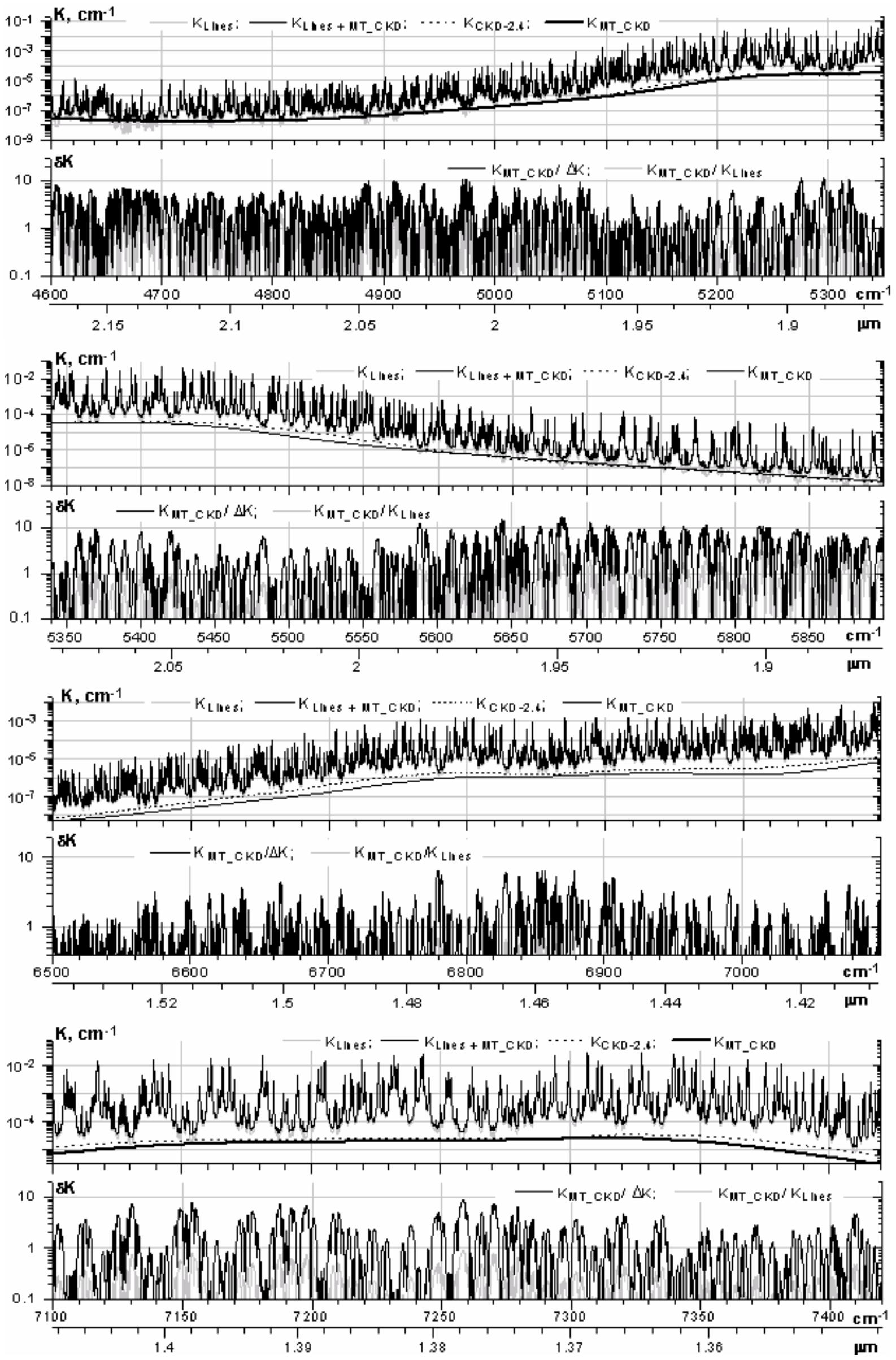
Fig. 6
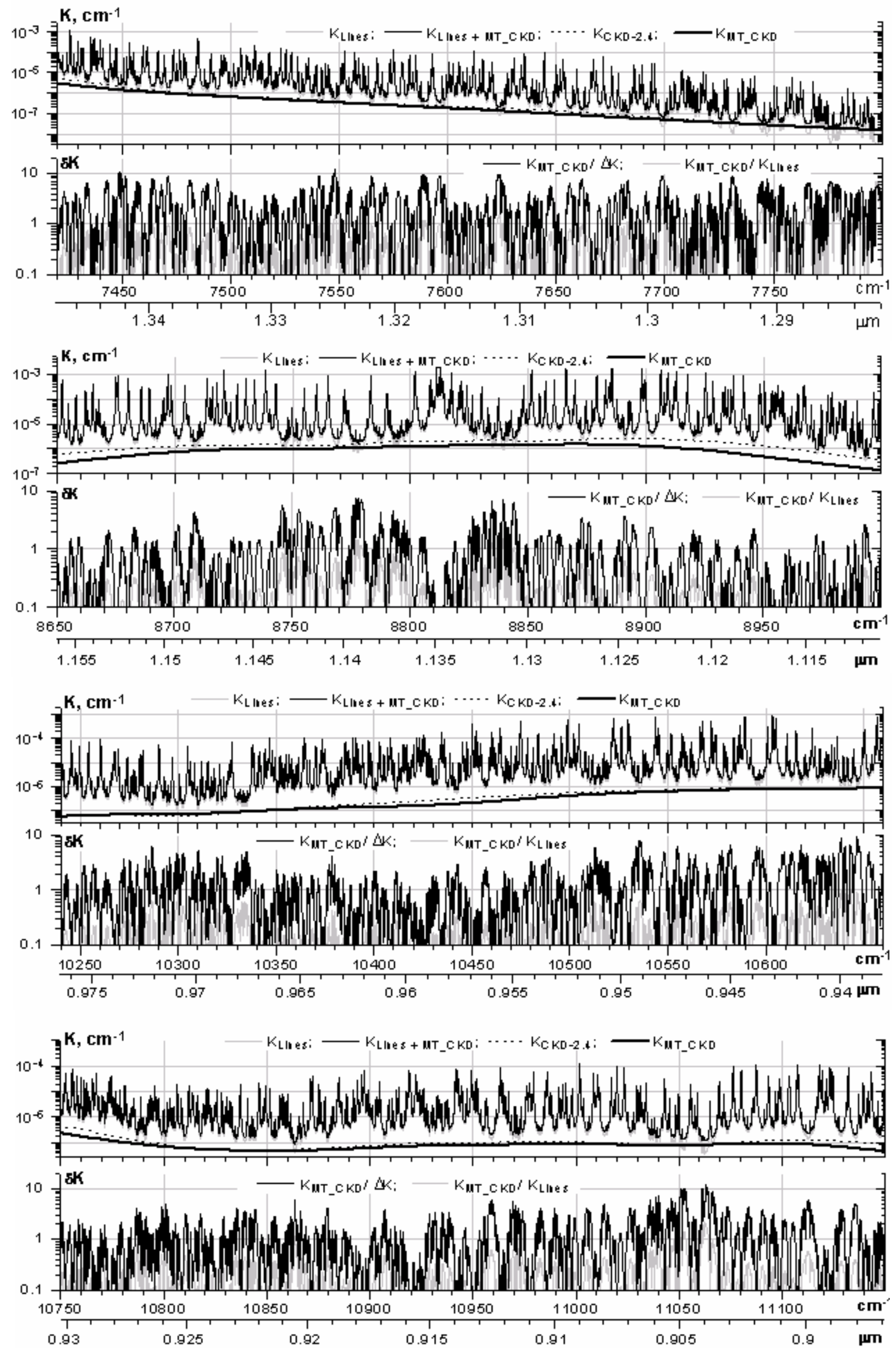
Fig. 7
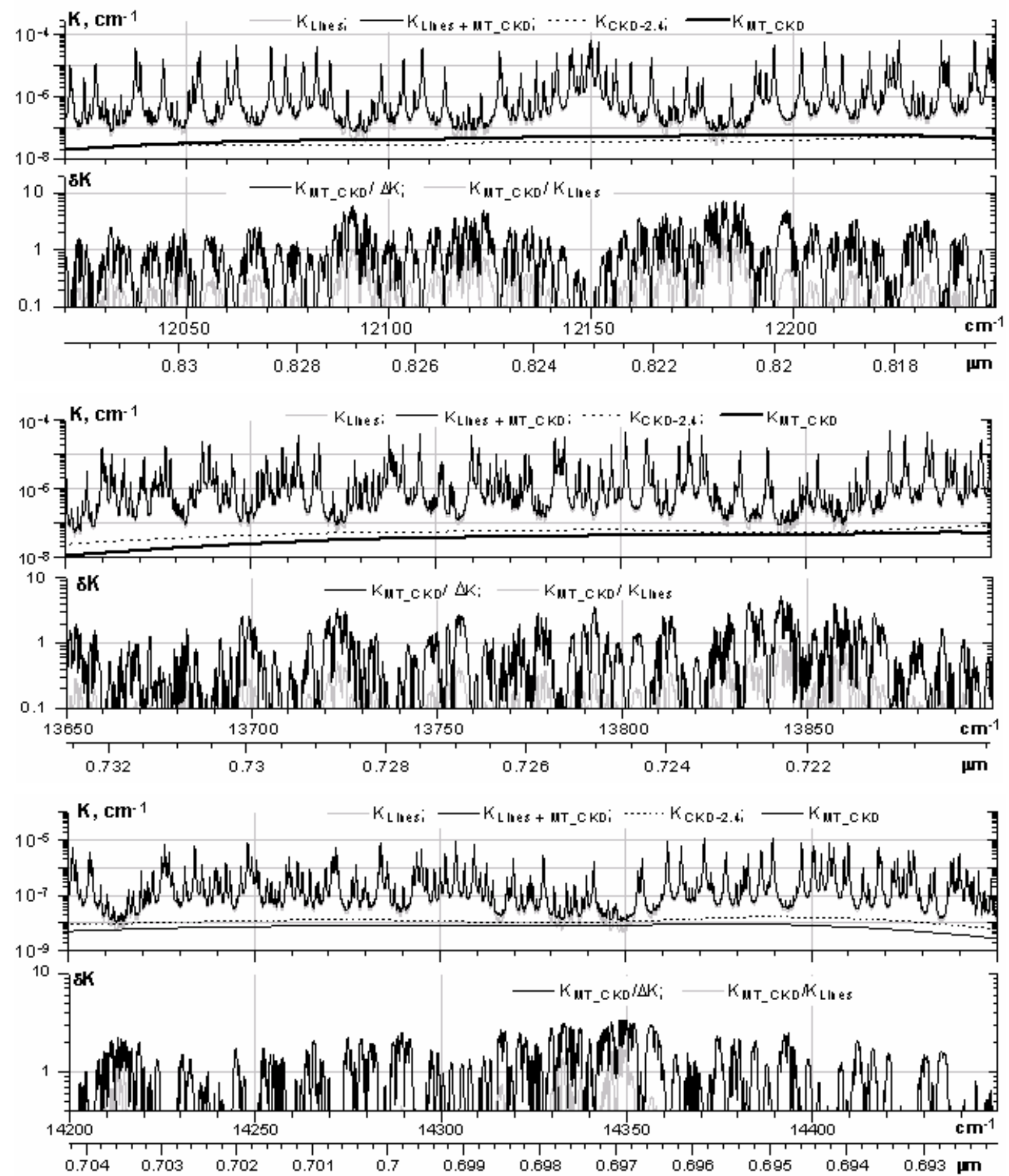
Fig. 8

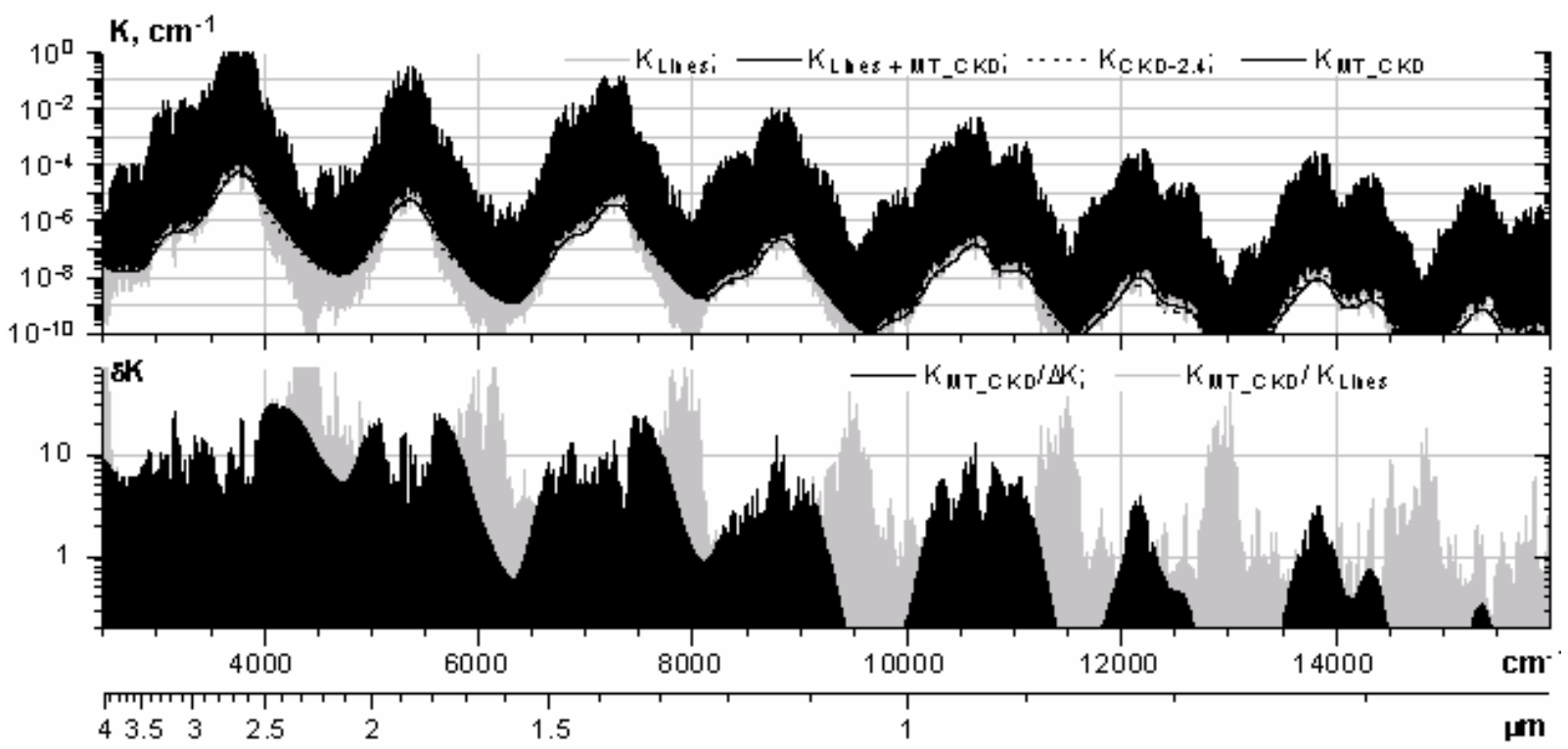


Fig. 9
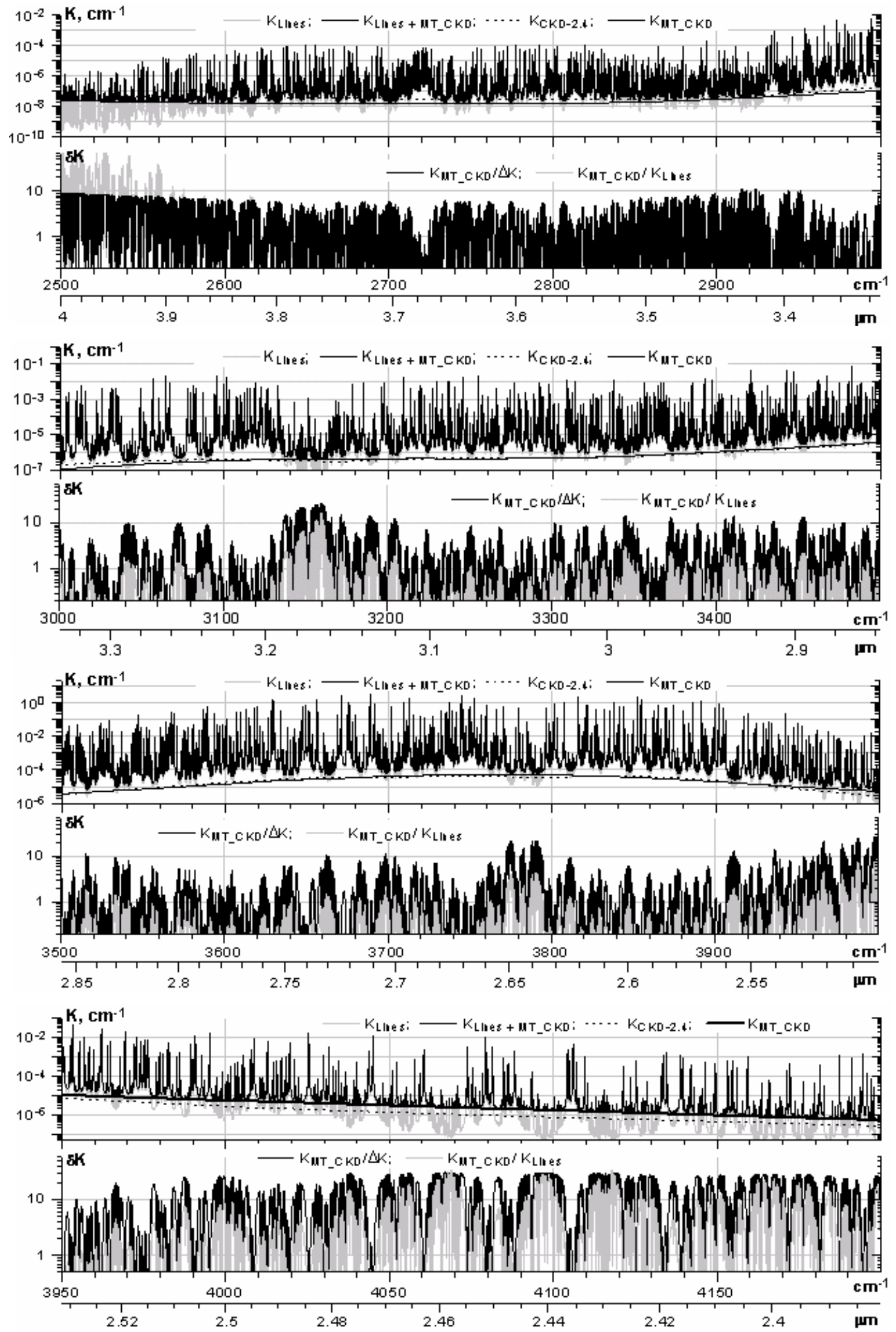
Fig. 10
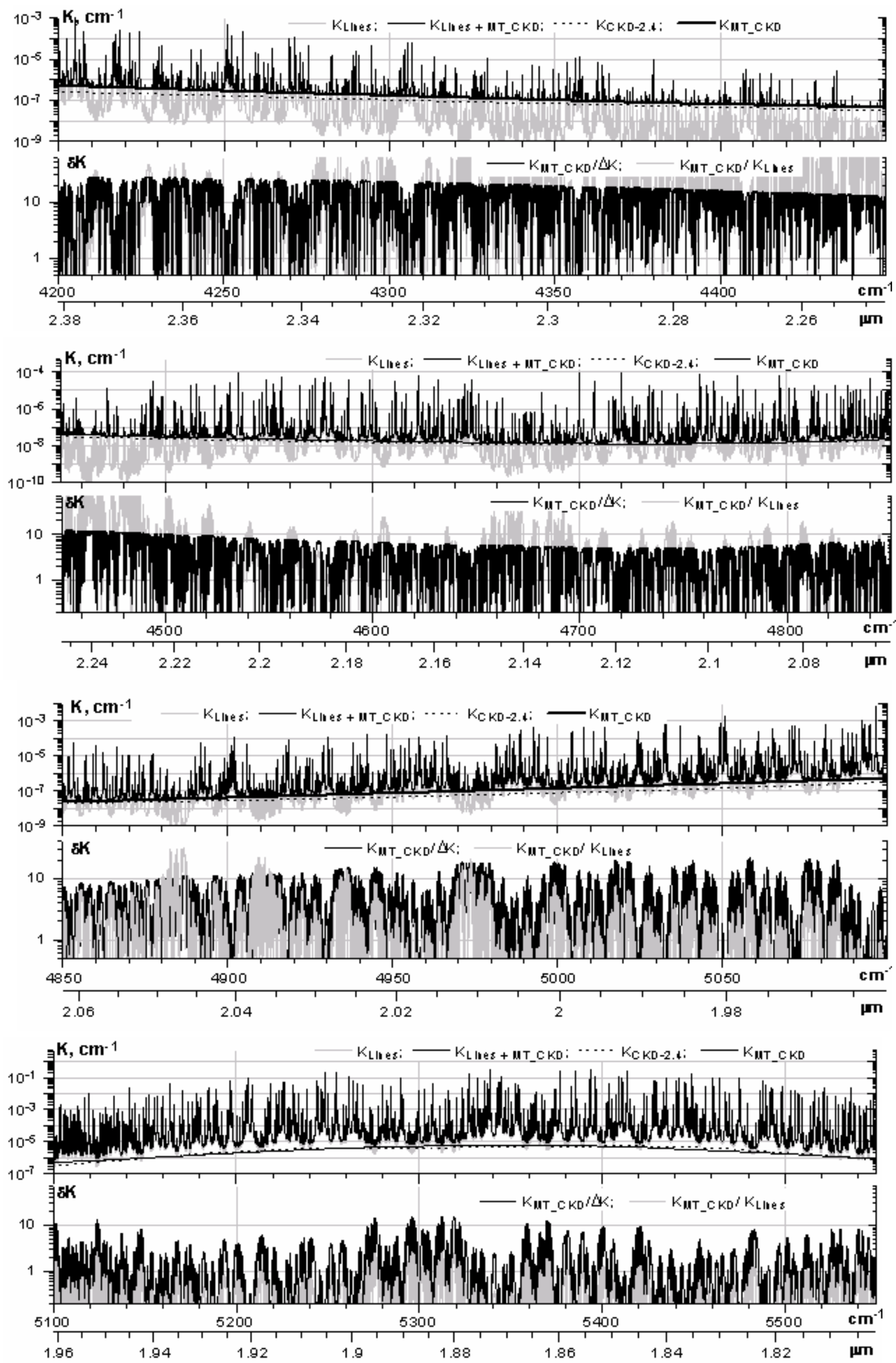
Fig. 11
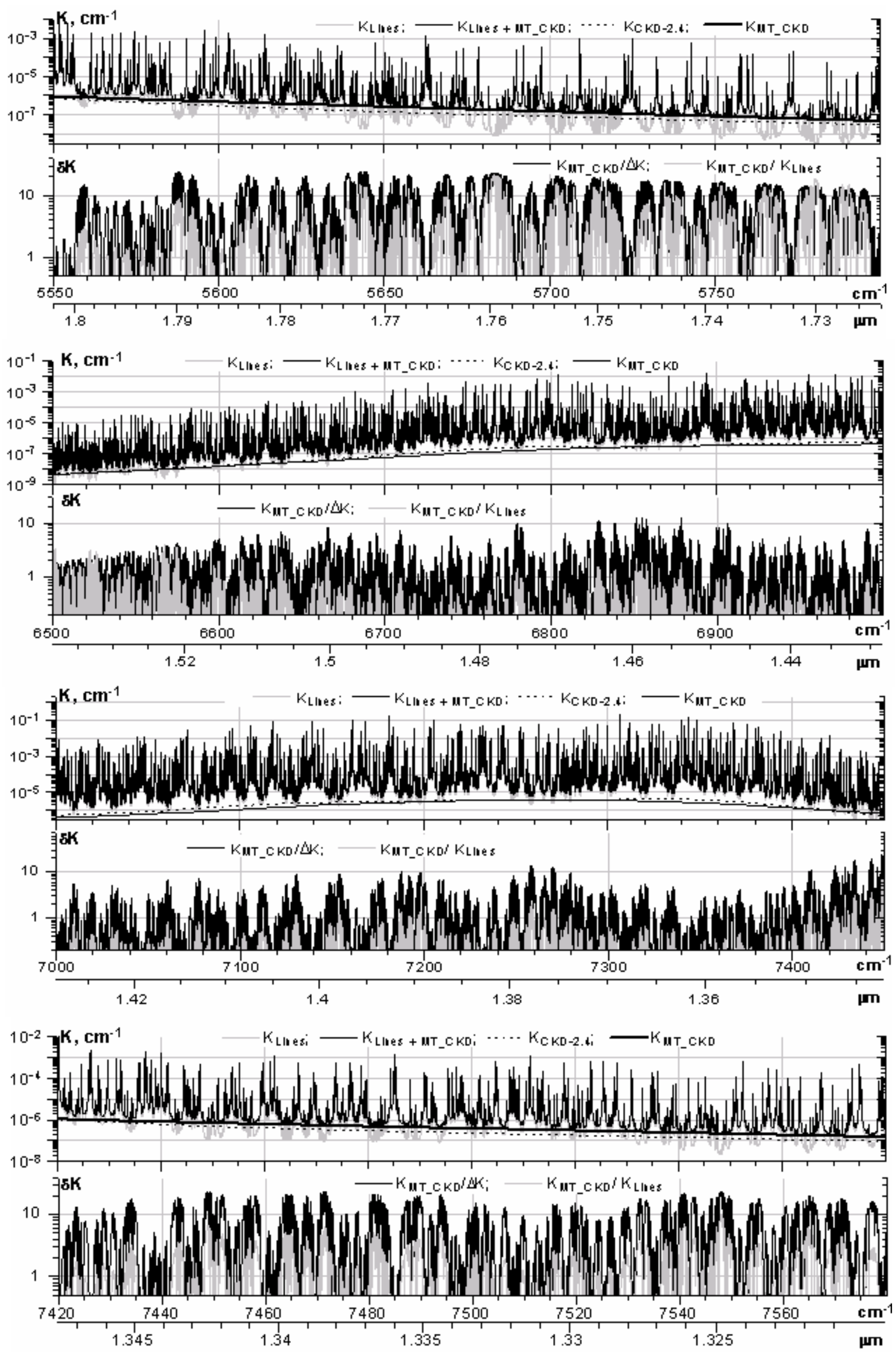
Fig. 12
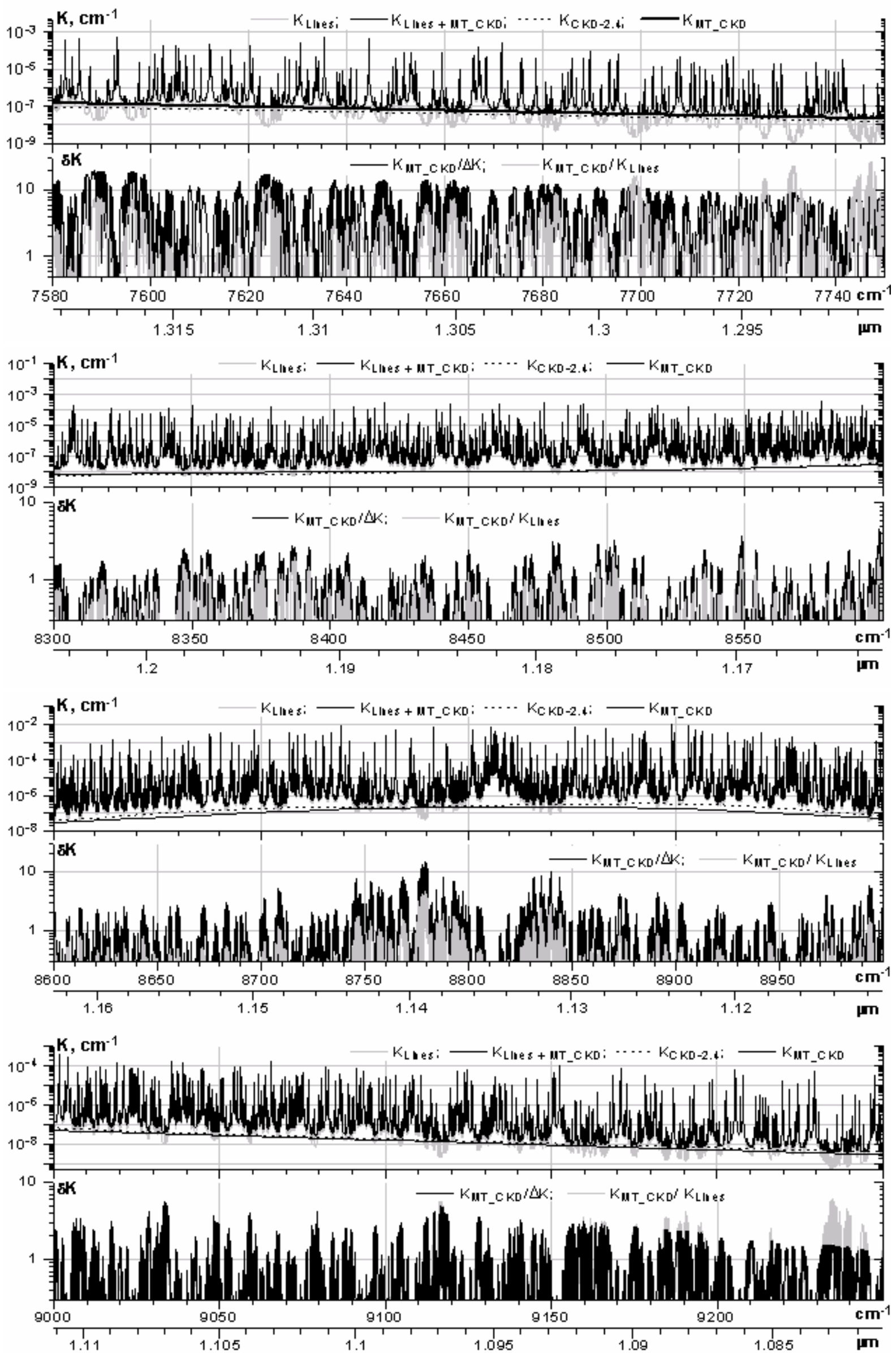
Fig. 13

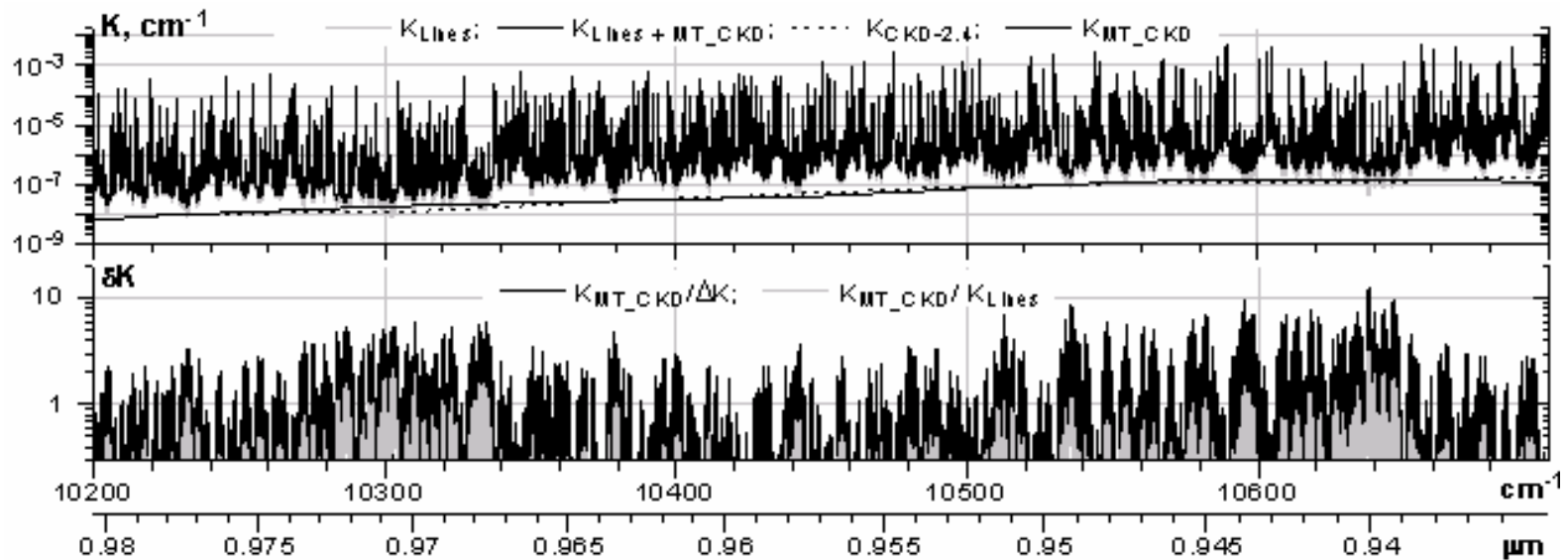

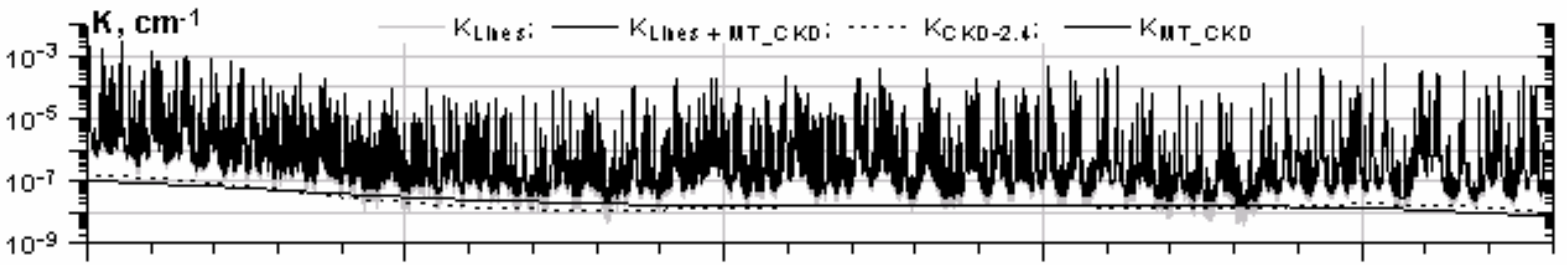
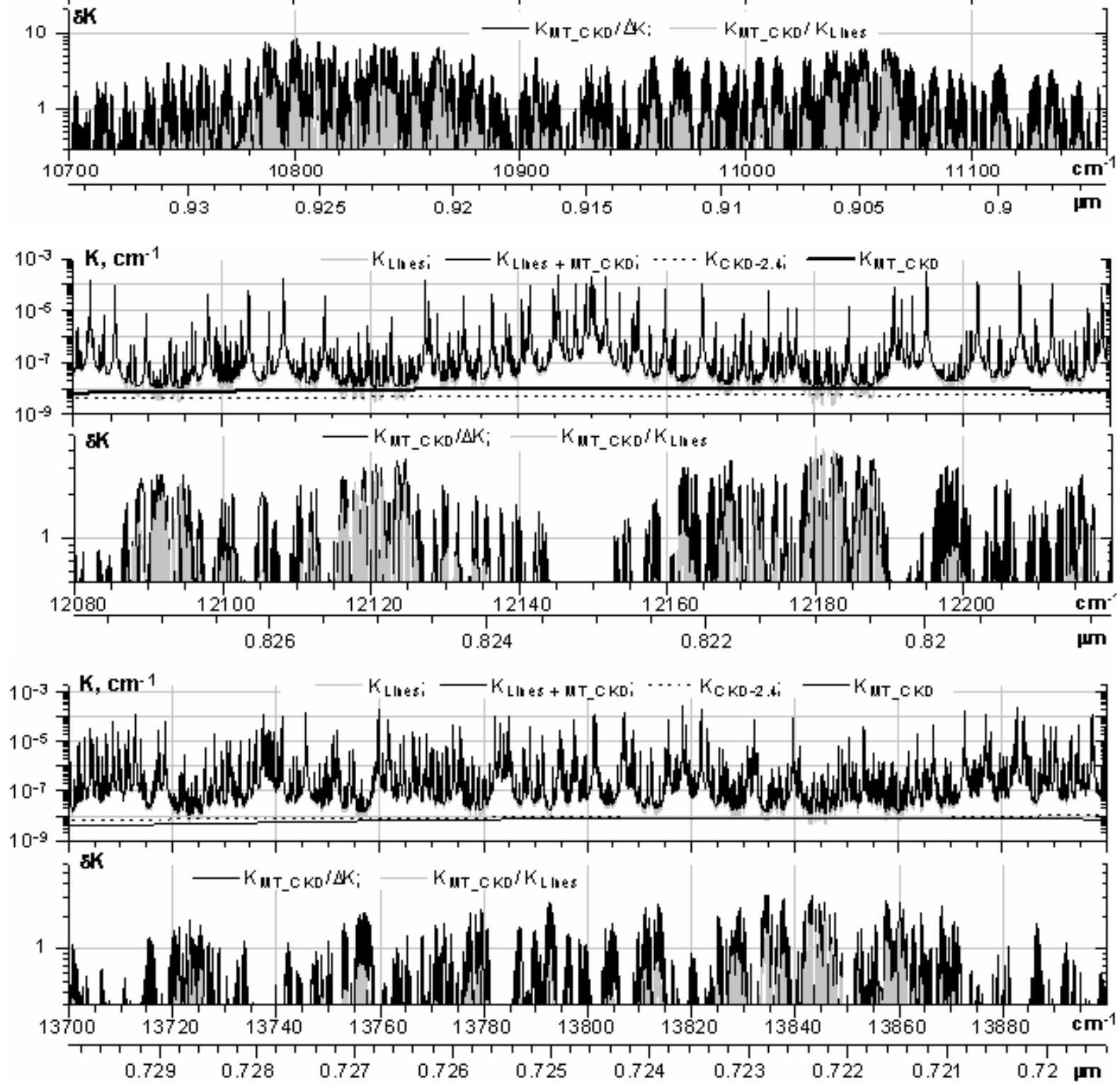